\title{
PROPOSTA DE UM MODELO DO SERVIÇO DE REFERÊNCIA DIGITAL PARA A OTIMIZAÇÃO DE BUSCA ÀS INFORMAÇÕES DISPONÍVEIS EM CATÁLOGOS DIGITAIS
}

\author{
PROPUESTA DE UN MODELO DE SERVICIO REFERENCIA DIGITAL \\ PARA LA OPTIMIZACIÓN DE LA BÚSQUEDA DE LA INFORMACIÓN \\ DISPONIBLE EN LOS CATÁLOGOS DIGITALES
}

\author{
Patrícia da Silva Moreno pattism@iq.com.br \\ Doutoranda em Ciência da Informação e Mestre em Ciência da Informação (Unesp) \\ Grupo de Pesquisa Novas Tecnologias em Informação (Unesp-FFC)
}

\begin{abstract}
Plácida Leopoldina V. A. da Costa Santos placida@marilia.unesp.br Doutora em Linguística. Professora de Pós-Graduação em Ciência da Informação (Unesp) Coordenadora do Grupo de Pesquisa Novas Tecnologias em Informação (Unesp-FFC)
\end{abstract}

\begin{abstract}
Resumo
O ambiente eletrônico para a disponibilização de informações vem incorporando com freqüência os serviços oferecidos pelas bibliotecas, procurando proporcionar facilidades na localização de informações e de documentos nas redes eletrônicas. Entretanto, muitos usuários encontram dificuldades na interação com certas interfaces, o que torna a busca por informações desestimulante e muitas vezes estressante. Com a aplicação do agente de interface busca-se avaliar a atuação deste tipo de software como facilitador da interação do usuário com acervos disponíveis, via catálogos digitais. E ainda verificar através do experimento com o protótipo, baseado na metodologia de avaliação heurística quais ações deverão ser tomadas para a correção dos erros que afetam a usabilidade da interface, além de confirmar a hipótese de eficácia de um sistema dessa natureza. Como resultado demonstra-se a construção de um sistema que utiliza ação interdisciplinar entre a Ciência da Informação e a Ciência da Computação na otimização de busca às informações disponíveis em catálogos digitais e na Internet com a ação de um agente de interface como mediador.
\end{abstract}

\section{Palavras-Chave}

Usabilidade de interfaces; Serviço de referência digital; Agentes de interface; Interação humanocomputador 


\section{INTRODUÇÃO}

Com o advento da Internet, as bibliotecas, em especial as universitárias, passaram a oferecer serviços de referência digital para servir de suporte aos usuários presenciais e aqueles fisicamente distantes, ou seja, usuários remotos.

A denominação Serviço de Referência - tomada da tradução de "reference work", baseado no latim referre, que significa indicar e informar, apareceu de forma impressa somente em 1886, mas o primeiro trabalho sobre o serviço de referência foi publicado em 1876 na primeira conferência da American Library Association (ALA), por Samuel Sweet Green que formulou a primeira proposta de um programa de assistência pessoal aos usuários de várias categorias que vê a biblioteca com diversos propósitos.

Segundo Macedo (1990), o Serviço de Referência (SR) em sentido amplo, é considerado a interface entre informação e usuário, tendo à frente o bibliotecário de referência, respondendo questões e auxiliando o usuário, por meio de conhecimentos profissionais. Para a autora o termo "atendimento" é alterado por "interface" com uma mudança de postura dos profissionais de referência quanto a sua forma de atuação: se antes bastava a mera indicação de materiais que poderiam conter a resposta para o pedido formulado pelo usuário, agora já se alerta para a adequação da necessidade desse usuário ao acervo da biblioteca.

Para Oliveira e Bertholino (2000), a Internet marca nitidamente a transformação entre SRs tradicionais (para o usuário presencial) para os SRs voltados para os usuários remotos. No SR tradicional o bibliotecário mantém controle e opera de forma independente. Com o usuário remoto a relação é diferente, pois ele controla o processo de acordo com sua conveniência, preservando anonimato, selecionando fontes, descartando e buscando outros SRs.

Em virtude do uso das novas tecnologias de informática a disponibilização da Biblioteca exige considerável atenção aos problemas de interação dos usuários com os sistemas de informação automatizados, adaptando os serviços e os produtos oferecidos às necessidades dos usuários. Diante desta natureza, o serviço de referência requer maior flexibilidade e amplitude de conhecimento por parte do bibliotecário. De acordo com Figueiredo (1999), a compreensão da complexidade dos vários sistemas de comandos e interfaces e o domínio dos elementos críticos e estruturas dos recursos eletrônicos de 
informação são os componentes essenciais do conhecimento básico dos bibliotecários de referência.

Para Márdero Arellano (2001, p.2) os serviços de referência digitais estão cada vez mais atuantes e há um avanço no interesse por essa área. Segundo o autor, "na Internet podem ser encontradas bibliotecas que oferecem serviço de referência em tempo real, por meio do acesso à base de dados, telefone, e-mail, formulários na web, videoconferência, 'Internet chat', páginas de FAQ ou mural'.

Além disso, podemos encontrar diversas vantagens com o uso do serviço de referência digital, tais como: busca em bases de dados on-line com respostas rápidas às questões dos usuários; uso do hipertexto e hipermídia na qual possibilita o usuário navegar por "infovias" mais amigáveis, de acordo com a sua escolha e em diferentes mídias; e agilidade nos acessos mais rápidos e com menos burocracia, diminuindo o tempo gasto pelo usuário e pelos bibliotecários.

Conforme as vantagens apresentadas, Bax (1998) ressalta que a Web é de importância fundamental para as bibliotecas e centros de informação. Aquelas bibliotecas que não forem capazes de integrar tais mudanças de forma efetiva, ainda que gradual, simplesmente desaparecerão ao longo do tempo, muito provavelmente por falta de usuários.

Embora a Internet ofereça grandes vantagens aos serviços oferecidos pela biblioteca, muitos usuários enfrentam problemas ao utilizar esses serviços, tais como: inaptidão para lidar com computadores, dificuldade em formular expressão de busca, utilização de interfaces de busca com diversas formas de geração de expressão de busca e formas de visualização avançadas, não-padronização dos diferentes sistemas de bibliotecas etc. Tais fatores tornam o processo de busca difícil e muitas vezes desestimulante. Pois a diversidade de opções de busca com comportamento e terminologia variada de um sistema para outro, textos extensos de ajuda ao usuário e interfaces pouco atrativos geram dificuldades na realização do serviço de busca à informação.

Quando determinado usuário acessa uma ferramenta de busca, ele freqüentemente tem uma compreensão confusa de como pode alcançar seu objetivo. Muitos dos usuários possuem pouco conhecimento em informática, desconhecem as possibilidades e estratégias de busca das ferramentas e utilizam um vocabulário limitado de sinônimos das palavras-chave sobre um determinado assunto, o que dificulta a compreensão do processo e o uso de sistemas de busca. 
Portanto, apresenta-se como proposta o desenvolvimento de uma interface gráfica, fornecendo estilos de interação, tais como, botões, diálogos baseados em perguntas e respostas, diálogo de preenchimento de formulário e diálogo em linguagem natural para possibilitar que o usuário não especialista possa interagir com o sistema na sua própria linguagem, no qual o agente de interface passará a ser o mediador na interação do usuário com o sistema, minimizando o problema de interação humano-computador com recursos informacionais em catálogos digitais. Tendo como hipótese um Serviço de Referência Digital que ofereça interfaces facilitadoras para o uso e acesso de informações disponíveis em bases de dados bibliográficos.

\section{APRESENTAÇÃo do PROTÓtiPo: ARQUiteTURA, MOdELAGEM E IMPLEMENTAÇÃO}

O Serviço de Referência Digital desenvolvido é um serviço de filtragem de informação em base de dados de recursos digitais disponíveis na Web que consiste na utilização da tecnologia de agentes a fim de proporcionar ao usuário uma interface capaz de atender as suas necessidades informacionais.

Cada usuário deste sistema tem uma personalidade única, ou seja, diferem em seus objetivos, experiências e conhecimento sobre um assunto. É por isso que o Serviço de Referência Digital utiliza-se do processamento de linguagem natural, onde o usuário terá acesso a uma "busca avançada" sem ter conhecimento das estratégias de busca. Além disso, buscamos projetar uma interface totalmente amigável com o uso da tecnologia de agentes, mas especificamente os agentes de interface e com os estilos de interação, no qual possibilite ao usuário reagir eficientemente às tarefas que são exigidas pela interação.

O acesso a catálogos eletrônicos de bibliotecas e unidades de informação na web é uma realidade. Através do Serviço de Referência Digital desenvolvido é possível realizar buscas em paralelo a diferentes provedores de informações. Para sua construção foi necessário levarmos em consideração alguns atributos de qualidade relativos à construção de um produto de software, tais como, estruturação, modularidade, legibilidade, concisão, simplicidade, distribuição dos elementos na tela e estilos.

O protótipo foi projetado não só com o objetivo de permitir aos usuários locais e externos um instrumento de pesquisa, mas também colocar disponível a comunidade de pesquisadores e estudiosos um instrumento ágil de difusão e intercâmbio do conhecimen- 
to. O sistema aqui proposto pode ser usado isoladamente (acessando diretamente a URL) ou como parte de um ambiente interativo na Internet.

$O$ agente de interface implementado (ver figura 1) exerce a função de mediador na forma de comunicação entre o sistema e o usuário. A interface onde o agente atuar é constituída de uma tela gráfica montada em um browser com componentes básicos de HTML para funcionar de forma irrestrita na Web. $O$ agente de interface interage com o usuário do sistema em linguagem natural, através de balões textuais, permitindo desta forma que o usuário também se comunique com o agente em linguagem natural e através da qual as buscas são realizadas.

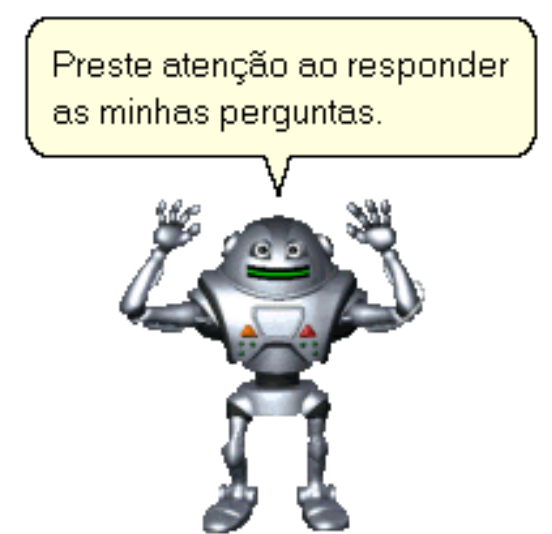

Figura 1 - Agente da Microsoft Robby

Fonte: Moreno (2005)

Através da interface, o usuário faz requisições na forma de sentenças em linguagem natural para o agente de interface. Depois de efetuado o processamento, a resposta é enviada ao usuário. Além do agente de interface ser o mediador entre o usuário e o computador, a sua missão é de ajudar na construção do modelo do usuário, no qual as informações contidas neste modelo servirão para adaptar a busca conforme as necessidades individuais do usuário. Outra missão atribuída ao agente é de processar as requisições recebidas pela interface aplicando as estratégias de busca adequadas para a escolha das respostas de acordo com as informações apresentadas pelo usuário.

Este protótipo disponibilizará o serviço de busca nas seguintes bases de dados: Base experimental, Google, nos acervos públicos como a Biblioteca Digital de Teses e Dissertações da USP, Biblioteca Digital da UNICAMP e do Portal de Bibliotecas da UNESP. Desta forma, o Serviço de Referência Digital visa dar uma visão geral dos textos disponíveis na rede como também nos catálogos digitais destas instituições, a fim de, possivelmente obter um resultado mais satisfatório. Entende-se como Base de dados experimen- 
tal, um banco de referências bibliográficas de uma Biblioteca $X$ modelada nesta pesquisa para fins de testes.

Para satisfazer as necessidades informacionais dos estudantes iniciantes ou veteranos, professores, pesquisadores, bibliotecários, e também a comunidade em geral, procurou-se fazer um planejamento cuidadoso da interface usuário e o serviço de referência digital com base no público que o sistema irá atingir. Levamos em consideração os seguintes aspectos para a modelagem do Serviço de Referência Digital:

- A homepage é composta de linguagem simples e vocabulário de seus usuários. Constando de um banner na parte superior da página (desenvolvido em Photoshop) apresentando um logotipo e o título da página, conforme a figura abaixo.

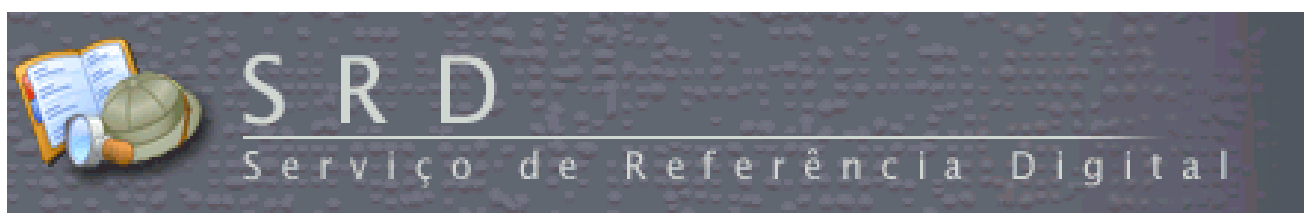

Figura 2 - Banner do Serviço de Referência Digital Fonte: Moreno (2005)

- Na parte inferior da homepage apresenta, a URL da página, o nome da coordenadora do sistema, endereço de e-mail e assinada pelo responsável por sua manutenção, a fim de que os usuários possam entrar em contato com o responsável pela edição e também para que aqueles que as imprimam possam lembrar de sua localização (ver figura 3). Além disso, é disponibilizado arquivo de programas para instalação do agente de interface caso não houver o ator instalado no microcomputador do usuário, como também, arquivos que possibilitam o agente se comunicar com o usuário através da fala. Esta estrutura permanece durante toda a interação do usuário com o sistema.

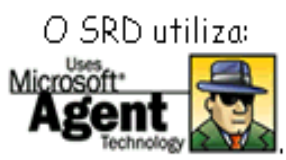

Se não puder ver o Robby, robổ Bibliotecário Digital, clique aqui para instalar o ator.

Para escutar o Robby falando, instale estes programas: spchapi.exe e lhttsptb.exe.

Coordenaçãa: Dra. Plácida L. V. A. Costa Santos

Desenvolvimento e Manutençã Patrícia Moreno - patriciamoreno@yahoo.com - URL:

www.cybercafeadmin.com.br/srd

Figura 3 - Descrição do sistema

Fonte: Moreno (2005)

- O emprego das cores na concepção do Serviço de Referência Digital tem como objetivo principal tornar a interface humano-computador rápida e agradável, a fim de não 
transmitir apenas informações, mas também possa chamar a atenção e associar objetos de interação. É necessário ressaltar que o uso de cores extras pode prejudicar na interpretação do usuário quanto ao serviço que está sendo oferecido. Segundo Bax (1998) um padrão de cores e formas consistentes é fundamental para que um site adquira identidade própria. O protótipo possui os padrões: fonte Comic Sans MS tamanho 14, cor de fundo lisa e o texto em preto, a fim de garantir um contraste adequado com os textos e rótulos. Em relação ao layout das telas que envolvem a fonte, formato de botões, cores e eventos foram mantidas consistências durante todo o protótipo, a fim de proporcionar conforto ao usuário, que localiza melhor as funcionalidades padrão se elas estão consistentemente colocadas na tela, na qual permitem que uma vez aprendido o estilo da interface do ambiente, o usuário possa se dedicar à aprendizagem dos conteúdos, sem se preocupar com a interface. É importante ressaltar que fontes de tamanho reduzido, cores de fundos confusos, textos móveis e longos prejudicam a leitura da informação apresentada. Enquanto o uso moderado destes recursos pode acrescentar interesse e motivação ao usuário que, ao invés de concentrar-se na informação que está sendo apresentada, irá se entreter com animações pouco relacionadas com a tarefa;

- o feedback e diálogos das ações do usuário diz respeito às respostas do sistema às ações do usuário. As ações praticadas pelo usuário no Serviço de Referência Digital podem ser um simples pressionar de botão, sentenças digitadas pelos usuários, até uma lista de itens a serem selecionados de acordo com a preferência do mesmo, portanto, as respostas do sistema a estas ações devem ser rápidas e precisas de acordo com a transação solicitada. Segundo Cybis (2003, p.32) a "qualidade e rapidez do feedback são dois fatores importantes para o estabelecimento de satisfação e confiança do usuário, assim como para o entendimento do diálogo, possibilitando que o usuário tenha um melhor entendimento do funcionamento do sistema."

O protótipo possui um único nível de acesso aos dados armazenados, entretanto, a interface possui a capacidade de buscar em mais de uma base de dados caso haja necessidade por parte do usuário. A linguagem natural é o meio pelo qual o usuário irá interagir com o Serviço de Referência Digital. Neste caso, a estratégia adotada pelo agente de interface no processamento da linguagem natural (PLN) consiste na metodologia de stopwords, ou seja, a eliminação das preposições, conjunções e artigos para estruturar os termos que serão submetidos ao sistema de busca. São eles: 


\section{Conjunções}

nem, não só, mas também, mas, porém, contudo, todavia, entretanto, no entanto, não obstante, ou, logo, portanto, por conseguinte, pois, que, porque, porquanto, se, uma vez que, visto que, já que, embora, ainda que, mesmo que, conquanto, apesar de que, caso, desde que, contanto que, como, conforme, consoante, segundo, de forma que, a fim de que, para que, à medida que, à proporção que, ao passo que, quanto mais, enquanto, antes que, depois que, logo que, assim que.

\section{Preposições}

a, após, ante, até, com, como, conforme, consoante, contra, de, desde, em, entre, exceto, fora, mediante, para, pra, pelos, pelas, pela, pelo, perante, por, salvo, segundo, sem, sob, sobre, senão, tirante, trás, visto.

\section{Artigos}

o, os, a, as, um, uns, uma, umas

Quadro 1 - Principais Conjunções, Preposições e Artigos.

Fonte: Cipro Neto e Infante (1998)

Contudo, procurou-se adotar o stopwords como método para a compreensão da linguagem natural, com o intuito de oferecer ao usuário não especialista uma ferramenta onde ele possa fazer consultas em sua própria língua. Desta forma, o usuário terá o mínimo de esforço mental possível com o uso deste sistema, o qual não necessitará ao usuário ter conhecimento sobre operadores booleanos e outras estratégias utilizadas para se conseguir realizar buscas eficientes.

Observando a figura 4, podemos exemplificar o funcionamento geral do protótipo Serviço de Referência Digital, traçando a seguinte descrição: 


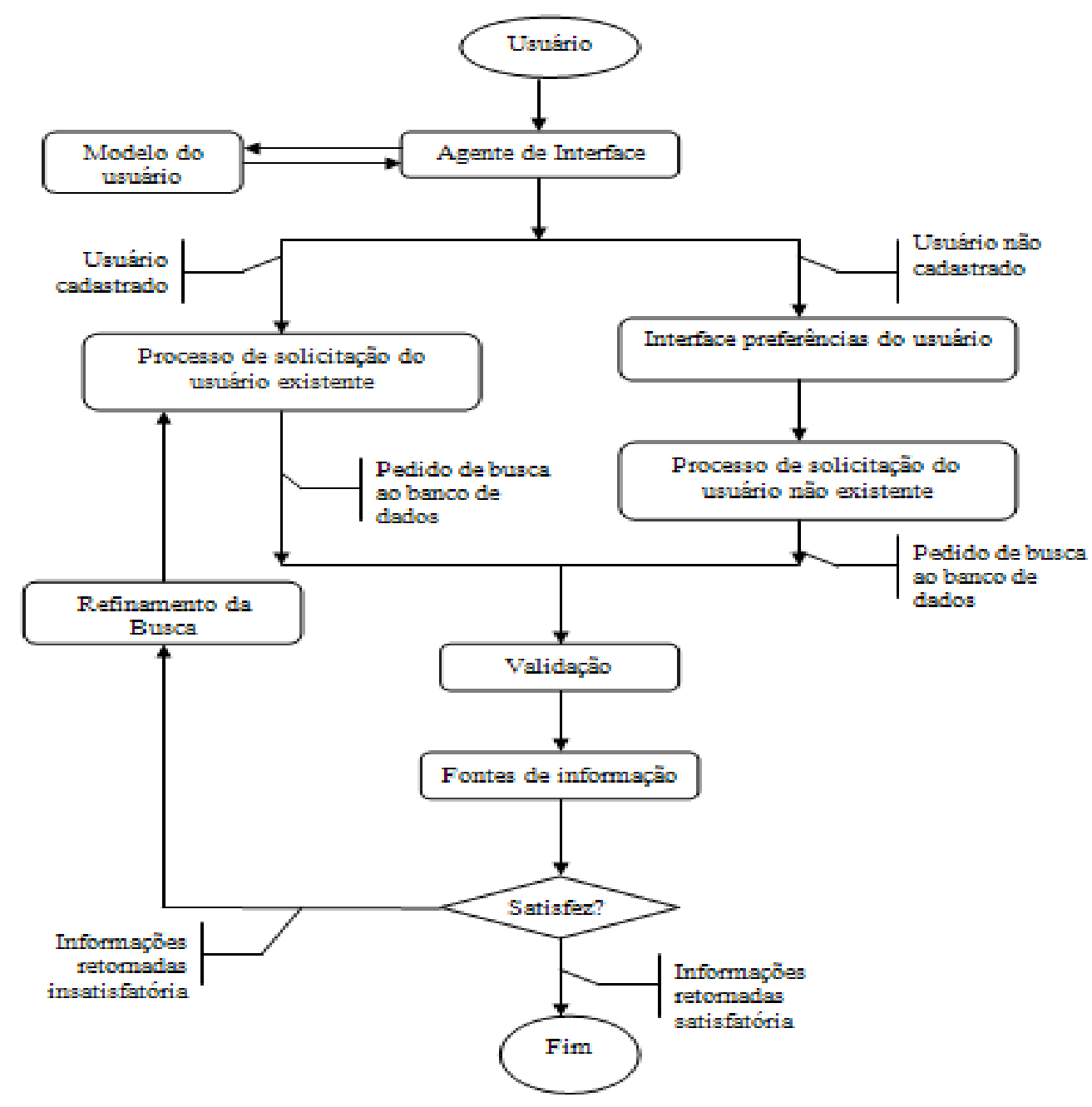

Figura 4- Funcionamento geral do Serviço de Referência Digital

Fonte: Moreno (2005)

1. O usuário acessa a homepage, preenchendo os campos nome do usuário e senha, passando pelo processo de autenticação.

2. Em seguida o agente de interface verifica se o usuário está armazenado no modelo do usuário. Caso afirmativo, o usuário é encaminhado para o processo de solicitação do usuário existente. De outra forma, o usuário é encaminhado para a interface Preferências do usuário, onde o usuário define as suas preferências de acordo com as suas necessidades. As informações coletadas do usuário irão compor o modelo do usuário, o qual, o agente de interface utilizará para a adaptação do resultado da busca. Logo após, o usuário é encaminhado para o processo de solicitação do usuário não existente. Observa-se que não foi utilizado o método baseado no IP da máquina para a identificação do usuário, visto que, geralmente, uma máquina é utilizada por vários usuários como também um mesmo usuário pode utilizar-se de várias máquinas. 
3. Antes de pesquisar na base de dados disponibilizados para esta ferramenta, o agente de interface valida os temas de interesse apresentados pelo usuário construindo a estratégia de busca. A tarefa de construir a estratégia de busca usando os operadores booleanos é embutida pelo agente de interface.

4. Depois de concluído a busca de acordo com os interesses do usuário, o resultado é apresentado em uma única janela. No caso da busca ocorrer em várias bases de dados ao mesmo tempo, o resultado será apresentado na forma de janelas sobrepostas, na qual cada janela corresponderá uma base de dados. Em seguida, o agente demonstra interesse em ajudar a solucionar o problema informacional do usuário, sugerindo o processo de refinamento da busca, para que se possa definir melhor o objeto de interesse e tornar a pesquisa mais eficiente.

Nesta pesquisa procurou-se desenvolver um Serviço de Referência Digital apresentando uma interface amigável, incluindo características relacionadas aos critérios de ergonomia de software. Para Lieberman (2002) uma interface é ergonômica quando as técnicas de construção de telas, de diálogo, de comunicação gráfica e visual, conduzem a comunicação homem-computador a um estado de perfeito entendimento, conforto e satisfação do usuário no uso de sistema computacional.

A interface proposta no protótipo do Serviço de Referência Digital é dividida em dois frames: um frame que contém o agente de interface e um frame para a apresentação do resultado. Delas derivam as demais interfaces e janelas do protótipo.

O sistema está implementado em HTML por ser uma linguagem básica para a criação de documentos hipertexto para a Web. Todas as páginas modeladas são estruturadas com essa linguagem por apresentar características ideais para o desenvolvimento do protótipo aqui proposto, tais como: portabilidade (texto em HTML é escrito usando apenas a tabela ASCII, funcionando bem em qualquer plataforma); flexibilidade (com o HTML, pode-se formatar textos de forma altamente elaborada, através de combinação de tags) e tamanho reduzido (seu código ocupa pouquíssimo espaço, que é altamente propício para a transferência de documentos através da Internet).

Foi também utilizado o PHP (Personal Home Page Tools) uma linguagem que permite criar sites WEB dinâmicos, possibilitando uma interação com o utilizador através de formulários, parâmetros da URL e links. Muitos adeptos do PHP estão surgindo devido às várias funcionalidades que se propõem, tais como: facilidade de programação, suporte a banco de dados, independência de sistema operacional e hardware, entre outros. Além do 
HTML e PHP, foram utilizadas também funções JavaScript para criação das interfaces de busca e ações do agente de interface. Cabe ressaltar que o servidor web utilizado para alocar o protótipo é do tipo Apache/1.3.27.

Este modelo consiste de informações estabelecidas pelo próprio usuário, a fim de obter com precisão do usuário os seus interesses e preferências e também por informações que o agente de interface captura durante a interação do usuário com o sistema no seu primeiro acesso, podendo ser alterado pelo usuário nos acessos futuros.

Uma vez estando na página do aplicativo, será carregada uma interface HTML com campos texto onde o usuário deverá entrar com os seus dados. A partir da tela principal do aplicativo (figura 5) derivam as demais interfaces e janelas do protótipo.

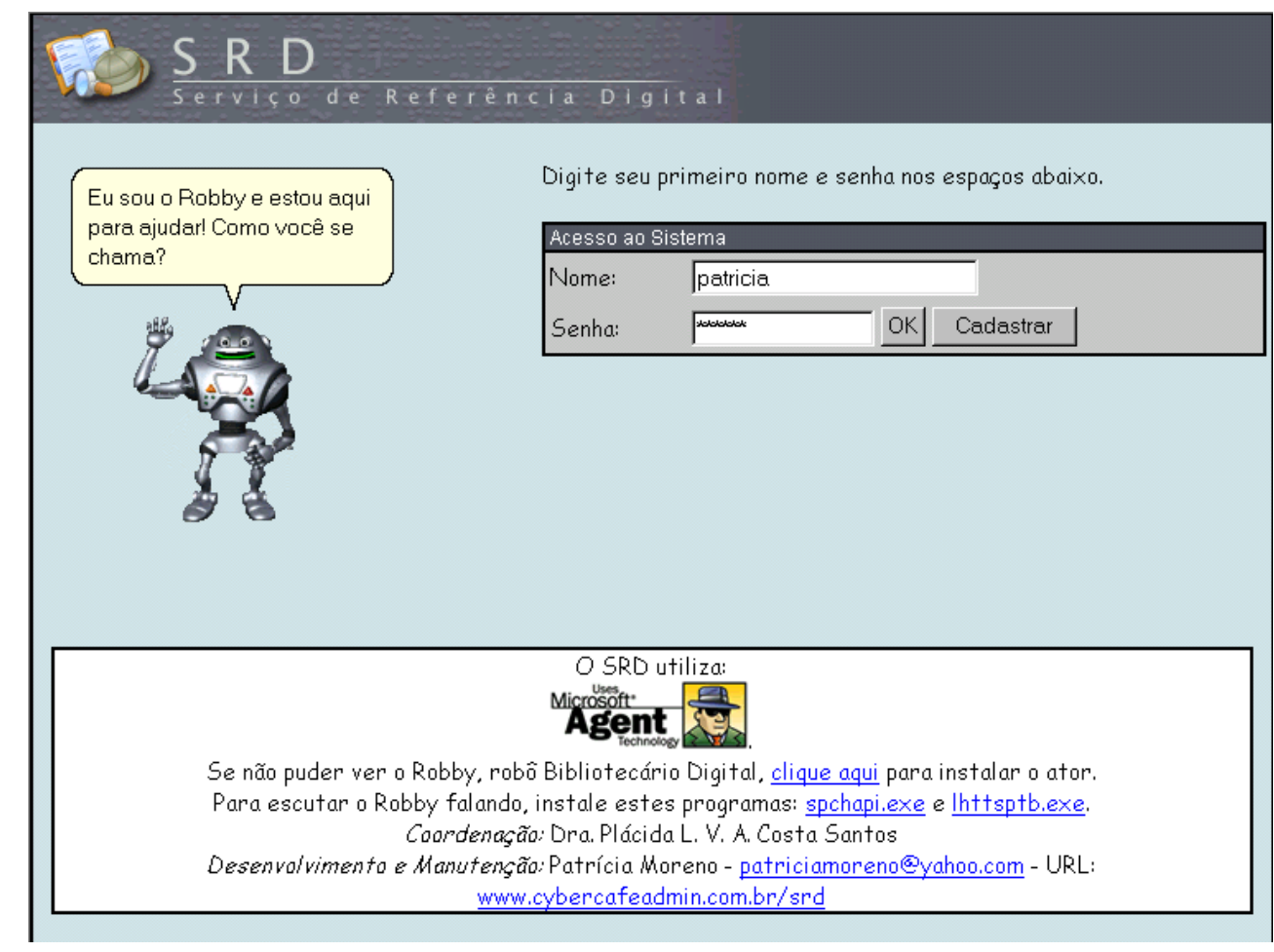

Figura 5 - Tela Principal do Serviço de Referência Digital Fonte: Moreno (2005)

Os links apresentados na tela principal serão utilizados caso o usuário do Serviço de Referência Digital não possuir o arquivo necessário para a utilização do agente de interface.

Podemos observar que a idéia desta pesquisa é dar sempre uma visão mais abrangente sobre o assunto pesquisado, tanto na rede quanto nos catálogos digitais destas instituições, a fim de economizar tempo destinado a pesquisa e recuperar informações mais satisfatórias. 
Devido aos problemas tais como, o desconhecimento do usuário no uso dos operadores booleanos e várias formas de interface de busca, buscamos nesta pesquisa desenvolver uma interface mais amigável no aspecto da interação do usuário com estes sistemas de busca. No Serviço de Referência Digital a tarefa de construir a estratégia de busca passará a ser de responsabilidade do agente de interface, ou seja, o usuário tem a tarefa apenas de responder as perguntas do agente de acordo com as suas necessidades informacionais. O uso dos operadores booleanos está embutido na atuação do agente de interface. Pode-se dizer que essa é uma das razões para se usar o Serviço de Referência Digital, além de fornecer os resultados de busca com rapidez e abrangência em várias unidades de informação com exatamente os termos solicitados, sem contar que é um meio fácil de interação para os vários perfis de usuário.

\section{RESULTADOS E DISCUSSÕES}

O desenvolvimento de sistemas computacionais interativos não pode ser considerado objeto de estudo apenas nas áreas de Ciência da Computação, da Engenharia de Software e de Fatores Humanos (ergonomia). A proposta desta pesquisa contribuirá para a área de Ciência da Informação fornecendo uma estrutura tecnológica para facilitar o projeto e implementação de interfaces que terá como objetivo o acesso otimizado aos recursos informacionais disponíveis em catálogos de bibliotecas na Internet e ainda possibilitar o melhor atendimento ao usuário, utilizando-se para tanto a tecnologia de agentes, proporcionando um ambiente computacional mais amigável. Além disso, tal ambiente proporcionará à comunidade um serviço de busca mais rápido às informações relevantes por meio de diálogo em linguagem natural e de interfaces gráficas que simplificam a interação do usuário com o computador, trazendo inovações e aplicações práticas na sua gestão e prestação de serviços.

Os usuários que não querem ou que não podem ir a biblioteca devido a problemas de deficiência, enfermidades, entre outras, serão grandes beneficiados com o desenvolvimento deste sistema que proporcionará a facilidade de acesso e interface amigável, para a conveniência e comodidade do próprio usuário.

Como vantagem para a comunidade acadêmica na implementação deste sistema destacamos os benefícios da apresentação dessa interface em uma homepage institucional como uma ferramenta de busca para diferentes catálogos digitais com acesso a grandes quantidades de informações e a redução de tempo destinado à pesquisa. 


\section{CONCLUSÕES}

A hipótese levantada nesta pesquisa é de um serviço de referência digital oferecendo interfaces facilitadoras para o uso e acesso de informações disponíveis em bases de dados bibliográficos. A partir disso, estudos foram realizados na área de Ciência da Computação, Ergonomia e Engenharia de Software, tendo como objetivo trazer contribuições destas áreas para a Ciência da Informação. E desta forma, aplicar os conceitos e desenvolver um serviço de referência digital.

Da Ciência da Computação trazemos a tecnologia de agentes inteligentes, da Ergonomia a teoria da interação humano-computador e da Engenharia de Software os atributos de qualidade relativos à construção de um produto de software, tais como, estruturação, modularidade, legibilidade, concisão, simplicidade, distribuição dos elementos na tela e estilos.

A utilização da tecnologia de agentes e dos conceitos da área de interação humanocomputador contribui para minimizar o problema de interação usuário e serviço de referência, além de proporcionar um ambiente amigável.

Embora o ambiente futurista que o agente de interface proporciona, é aconselhável o desenvolvimento de uma base de conhecimento, a fim de proporcionar capacidade ao agente de responder qualquer tipo de pergunta solicitada pelos usuários, e ainda, abordando alguns dos métodos do processo de compreensão da linguagem natural, tais como: Análise Morfológica, Análise Sintática, Análise Semântica, Discurso, Análise Pragmática, Fonológico e Lexical para auxílio no processo de interpretação e geração das sentenças.

Deixando de possuir uma arquitetura deliberativa, em que os agentes atuam com pouca autonomia e passando a ter uma arquitetura reativa, na qual não utiliza nenhum tipo de modelo ou raciocínio simbólico interno de seus ambientes.

Profissionais como, bibliotecários, webdesigner, entre outros devem se esforçar para o fornecimento de informação para todas as comunidades. Diante disso, um fator preponderante nesta pesquisa é referente aos elementos éticos envolvendo o ambiente web, a tecnologia de agentes e os fornecedores de informação.

Segundo Buchanan (1999) os fornecedores de informação mantêm grande poder e responsabilidade ética, pois, eles devem preservar valores culturais e especificidade, enquanto oferecer os melhores serviços àqueles que necessitam deles. 
Além das características descritas no decorrer do texto, o Serviço de Referência Digital tem como objetivo estar atento a sua contribuição no sentido de evitar ou amenizar problemas da sua comunidade, oferecendo sua ajuda na disponibilidade de informação para a redução de danos proporcionando maiores benefícios para a comunidade.

As aplicações que utilizam a tecnologia de agentes vêm fornecendo grandes benefícios para seus usuários, porém, trazem alguns problemas. No caso da Web o uso desta tecnologia pode causar sobrecargas em servidores e o aumento no tráfico em geral. Diante disso, devemos ficar atentos aos elementos éticos relacionados à tecnologia de agentes durante o seu desenvolvimento.

Vale ressaltar 3 normas éticas que foram levadas em consideração no desenvolvimento do agente de interface modelado nesta pesquisa. Tais como:

- os desenvolvedores devem obter clareza da utilidade e do funcionamento do agente antes de começarem a escrever, a fim de, evitar o consumo excessivo de recursos;

- desenvolvedores devem ter em mente que seus agentes poderão percorrer vários nós da Web, sendo que alguns podem não gostar da idéia de estar sendo visitados pelo seu agente. Segundo Koster (1996) neste caso, os desenvolvedores devem fazer uso dos processos de autorização, evitando maiores complicações futuras. Para Eichmann (1994) um agente deve respeitar as restrições impostas pelos operadores dos servidores aos quais ele obtém acesso, e não tentar perfurá-las. Independente do local visitado pelo agente, ele deve deixar da mesma forma como o encontrou. Cabe ressaltar que o agente de interface implementado nesta pesquisa não necessitou do uso dos processos de autorização, visto que, para acessar os catálogos de bibliotecas disponíveis na Internet e no índice Google, não foi necessário o acesso ao banco de dados;

- os desenvolvedores de agentes devem fazer testes localmente antes de colocarem seu agente para percorrer a Web, evitando assim a sobrecarga na rede.

Os projetistas devem ter a responsabilidade de refletir no seu próprio trabalho sobre este assunto anteriormente discutido, para evitar representação errada da missão e do serviço que está sendo oferecido, ou seja, responsabilidade em tomar precauções apropriadas e garantir que erros de modelagem não ocorram, especialmente quando os riscos são grandes (por exemplo, apresentação de informações duplicadas, falta de sigilo na 
entrega de informação ao usuário), e responsabilidade para informar os usuários se tais erros ocorrem e como podem ser solucionados.

\section{REFERÊNCIAS}

BAX, M. P. As bibliotecas na web e vice-versa. Perspectivas em Ciência da Informação, Belo Horizonte, v.3, n.1, p.5-20, 1998.

BUCHANAN, E. A. An overview of information ethics issues in a world-wide context. Ethics and Information Technology, n.1, p.193-201, 1999.

CIPRO NETO, P.; INFANTE, U. Gramática contemporânea da língua portuguesa. São Paulo: Scipione, 1998.

CYBIS, W. A. Engenharia de usabilidade: uma abordagem ergonômica. LABIUTIL, Laboratório de Utilizabilidade de Informática, Florianópolis, 2003.

EICHMANN, D. Ethical web agents. In: INTERNATIONAL WWW CONFERENCE, 2.,1994. Proceedings.... Disponível em:

<http://www.ncsa.uiuc.edu/SGD/IT94/Proceedings/Agents/eichmann.ethical/eichmann.htm l>. Acesso em: 25 abr. 2004.

FIGUEIREDO, N. M. Paradigmas modernos da Ciência da Informação. São Paulo: Polis; 1999. 168p. (Coleção Palavra-Chave, 10).

KOSTER, M., "WWW robot frequently asked questions". 1996. Disponível em:

<http://info.webcrawler.com/mak/projects/robots/faq.html>. Acesso em: 29 mar. 2004.

LIEBERMAN, H. Computer-aided design of user interfaces by example. In:

CONFERENCE ON COMPUTER-AIDED DESIGN OF USER INTERFACES, 2002, Valenciennes, France. Proceedings... Valenciennes, 2002.

MACEDO, N. D. Princípios e reflexões sobre o serviço de referência e informação. Revista Brasileira de Biblioteconomia e Documentação, São Paulo, n.1, v.23, p. 9-37, jan./dez, 1990.

MÁRDERO ARELLANO, M. A. Serviços de referência virtual. Ciência da Informação, Brasília, v.30, n.2, p.7-15, maio/ago. 2001.

MORENO, P. S. Serviço de referência digital: uma análise apoiada em agentes de interface. 2005. 153 f. Dissertação (Mestrado em Ciência da Informação) - Faculdade de Filosofia e Ciências, Universidade Estadual Paulista, Marília, 2005.

OLIVEIRA, N. M.; BERTHOLINO, M. L. F. Usuários remotos e serviços de referência (sr(s)) disponíveis nas home pages das bibliotecas universitárias. 2000. Disponível em: <http://snbu.bvs.br/snbu2000/docs/pt/doc/t013.doc. >. Acesso em: 12 mar. 2004. 


\title{
Title
}

The proposal of a digital reference service model to optimize the information search in digital catalogs

\begin{abstract}
The electronic environment for the dispose of information is frequently incorporating the services offered by the libraries, aiming to provide means to find information and documents in electronic networks. However, many users have difficulties in interacting with certain interfaces, which turns the search for information uninteresting and, most of the time, stressful. With the application of the interface agent the author intends to evaluate the performance of this type of software as a facilitator of the user's interaction with available collections, through digital catalogs. As well as to verify through experiments with the prototype, based on the heuristic evaluation methodology, which actions should be taken for the correction of mistakes that affect the interface usability, confirming the hypothesis of effectiveness of a system of that nature. As a result, the construction of a system that uses interdisciplinar actions between the Information Science and the Computer Science is demonstrated in the optimization of the search to the information available in digital catalogs and on Internet with the use of an interface agent as a mediator.
\end{abstract}

\section{Keywords}

Interface usability; digital reference service; interface agent; human-computer interaction

\section{Título}

Propuesta de un modelo de Servicio Referencia Digital para la optimización de la búsqueda de la información disponible en los Catálogos Digitales

\section{Resúmen}

El ambiente electrónico para la disponibilización de informaciones viene agregando con frecuencia los servicios ofrecidos por las bibliotecas, buscando facilidades en la localización de las informaciones y de los documentos en las redes electrónicas. Sin embargo, muchos usuários encuentran dificultades en la interacción con determinadas interfaces, lo que implica en una búsqueda poco estimulante y muchas veces estresante. Con la aplicación de una interfaz se busca evaluar la atuacción de ese tipo de software como facilitador de la interacción del usuario con los acervos disponibles, a través de los catalogos digitales. Así, se puede identificar, por médio del protótipo, con base en la metodología heurística, cuales acciones tendrán que ser desarolladas para la corrección de los errores que afectan la usabilidad de la interfaz. Además de eso, confirmar la hipótesis de la eficacia de un sistema de esa naturaleza. Resulta todavia en la construcción de un sistema que utiliza la acción interdisciplinar entre la ciencia de la información y la informática para optimizar la búsqueda de informaciones disponibles en los catálogos digitales y en la internet por medio de una interfaz mediadora.

\section{Palabras clave}


Usabilidad de las interfaces; servicio de referencia digital; agentes de la interfaz; interacción hombre-máquina; interacción humano-ordenador

Recebido em: 06.11.2008

Aceito em: 04.08.2009 